\title{
DNA polymorphism at the ACAULIS5 locus of the wild plant Arabidopsis thaliana
}

\author{
Kentaro Yoshida, Taku Kamiya, Akira Kawabe, and Naohiko T. Miyashita* \\ Laboratory of Plant Genetics, Graduate School of Agriculture, Kyoto University, \\ Sakyo-ku, Kyoto, 606-8502 Japan
}

(Received 17 September 2002, accepted 24 December 2002)

\begin{abstract}
Nucleotide variation in the ACL5 gene region, which encodes spermine synthase, was analyzed for 21 Arabidopsis thaliana ecotypes and one accession of Arabis gemmifera. In A. thaliana, dimorphism was also detected in the ACL5 region, as in other nuclear genes of this plant. The nucleotide diversity $(\pi)$ of the entire region, exon and intron was $0.0163,0.0042$ and 0.0293 , respectively. The level of nucleotide variation in this region was among the highest of those reported for genes in this plant species. The neutrality tests of Tajima, and Fu and Li did not detect significant deviation from test assumptions for the polymorphism data. However, the HKA test indicated that the level of polymorphism in the intron was significantly high, compared with $A$. gemmifera. The high nucleotide variation in the intron is responsible for the high level of nucleotide variation in the entire region. These results can be explained by elevated mutation rate in the ACL5 region in the A. thaliana lineage after the two species were split.
\end{abstract}

Key words: ACAULIS5, Arabidopsis thaliana, polymorphism, divergence

\section{INTRODUCTION}

Because plants have no nervous system, they have developed other sensing and control systems. Plants monitor a wide range of exogenous and endogenous signals and generate appropriate developmental responses to them (Gilroy and Trewavas, 2001). In plants, the hormone auxin is one of the most important endogenous signals for regulating growth and development (Gilroy and Trewavas, 2001). Auxin regulates the expression of many genes (Leyser, 2001), including ACAULIS5 (ACL5), a gene in A. thaliana which encodes spermine synthase, a protein composed of 339 amino acids. Inactivation of ACL5 causes a defect in the elongation of stem internodes by reducing cell expansion (Hanzawa et al., 2000). The internode elongation from the vegetative to reproductive stages is an important element in the A. thaliana life cycle. The number of adventitious roots formed in response to exogenous auxin differs significantly with the ecotype of A. thaliana (King and Stimart, 1998). Spermine synthase catalyzes the pathway that converts spermidine into spermine. Spermine is a kind of polyamine, which is involved in a wide range of processes in plants, such as triggering organogenesis and protecting against stress (Walden et al., 1997). The upstream region of the

Edited by Fumio Tajima

* Corresponding author: E-mail: arabis@kais.kyoto-u.ac.jp.
ACL5 gene contains putative auxin-responsive cis-acting elements (Hanzawa et al., 2000).

After investigating DNA variation of thirteen loci in $A$. thaliana (Miyashita et al., 1996; Innan et al., 1996; Kawabe et al., 1997; Kawabe and Miyashita, 1999; Purugganan and Suddith, 1998; Caicedo et al., 1999; Purugganan and Suddith, 1999; Stahl et al., 1999; Kawabe et al., 2000; Kuittinen and Aguadé, 2000; Aguadé, 2001; Hauser et al., 2001; Miyashita, 2001), two unexpected features of DNA polymorphism have emerged. One is the high level of DNA variation at the species level, despite the selfing nature of this plant. The average nucleotide diversity $(\pi$ : Nei and Li, 1979; Tajima and Nei, 1984) is estimated to be $0.74 \%$. This value is higher than that of Drosophila melanogaster (0.4\%: Moriyama and Powell, 1996). The other is a dimorphic pattern of DNA variation. In most loci, two divergent sequence types were detected. The presence of divergent sequence can account for the species polymorphism, depending on the frequency of the sequence types. The origin and maintenance mechanism of the dimorphism have been explained by three hypotheses. One is that two isolated populations of $A$. thaliana were fused before this plant species had expanded its habitat around the world (Innan et al., 1996). The second is introgression from another species. These two hypotheses do not involve natural selection. The other hypothesis is balancing selection. 
Strong support for overdominance has been obtained for Adh and PgiC (Innan et al., 1996; Kawabe et al., 2000; Miyashita, 2001). However, the exact cause and origin of the dimorphism remains to be clarified.

In this study, the level and pattern of DNA polymorphism in the 2.3-kb region of the ACL5 locus was analyzed for $A$. thaliana and $A$. gemmifera to elucidate the genetic mechanism involved in maintaining the DNA variation in these plant species. The genes whose expressions are regulated by auxin have not been investigated yet. Therefore, ACL5, which, influences plant elongation, might possess DNA variations related to the phenotypic variation caused by auxin. Another purpose was to elucidate the genetic mechanism involved in maintaining the nucleotide variation in the ACL5 region of $A$. thaliana. The study of additional regions such as the ACL5 region might contribute to determining the cause and origin of dimorphism.

\section{MATERIALS AND METHODS}

Plant material. Twenty-one ecotypes of A. thaliana of various origins were used (Table 1). For interspecific comparison, one A. gemmifera accession sampled at Ash- ibi, Kyoto, Japan, was used. The growth conditions for A. thaliana were as described previously (Kawabe et al., 2000).

DNA Extraction, PCR Amplification, and Sequencing. Genomic DNA was extracted from leaves with a modification of the CTAB procedure (Weising et al., 1991) and used for PCR amplification. A 2.3kb fragment of ACL5 region, which is located on the short arm of chromosome 5, was amplified. PCR primers were designed based on Columbia ecotype sequence (GenBank accession AF181093). Primers for PCR amplification were 5'ACCGAACCAAAGTAATCAAG-3', and 5'-ACGATGAGGAAGTGTTGAAA-3', located in the $5^{\prime}$ and $3^{\prime}$ flanking regions of $A C L 5$, respectively. The ACL5 sequence of 17 ecotypes of $A$. thaliana was determined by direct sequencing of PCR products. The PCR products of the remaining three ecotypes of $A$. thaliana (Bl-1, Bus-0, Edi-0) and one accession of $A$. gemmifera (Ashibi56) were cloned into the plasmid pUC18. The plasmid DNA was used as template for the sequencing reaction. The sequencing reaction was conducted by using the Cy5 Thermo Sequenase Dye terminator kit and the Thermo Sequenase fluorescent labeled cycle sequencing kit with 7-deaza-dGTP

Table 1. Plant materials analyzed in this study

\begin{tabular}{|c|c|c|c|}
\hline Species & Name code & Accession code & ${ }^{\mathrm{a}}$ Origin \\
\hline \multirow[t]{21}{*}{ Arabidopsis thaliana } & Col-0 & JA58 & United States \\
\hline & Dra-0 & JA68 & Czechoslovakia \\
\hline & Rou-0 & JA200 & Italy \\
\hline & Shokei & JW101 & Japan \\
\hline & Ws-0 & JA252 & Wh,Russia \\
\hline & $\mathrm{Ag}-0$ & JA2 & France \\
\hline & Nok-4 & JA170 & Netherlands \\
\hline & $\mathrm{Su}-0$ & JA225 & United Kingdom \\
\hline & Ts-1 & JA230 & Spain \\
\hline & In-0 & JA110 & Austria \\
\hline & Pog-0 & JA192 & Canada \\
\hline & $\mathrm{Mr}-0$ & JA155 & Italy \\
\hline & Hau-0 & JA100 & Denmark \\
\hline & Ita-0 & JA112 & Morocco \\
\hline & Bl-1 & JA13 & Italy \\
\hline & Kas-1 & JA119 & India \\
\hline & Edi-0 & JA69 & United Kingdom \\
\hline & Ost-0 & JA179 & Sweden \\
\hline & Bus-1 & JA46 & Norway \\
\hline & $\mathrm{Ci}-0$ & JA54 & United Kingdom \\
\hline & Cvi-0 & JA60 & Cape Verde Islands \\
\hline Arabis gemmifera & Ashibi56 & & Kyoto, Japan \\
\hline
\end{tabular}

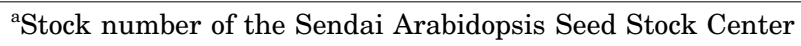




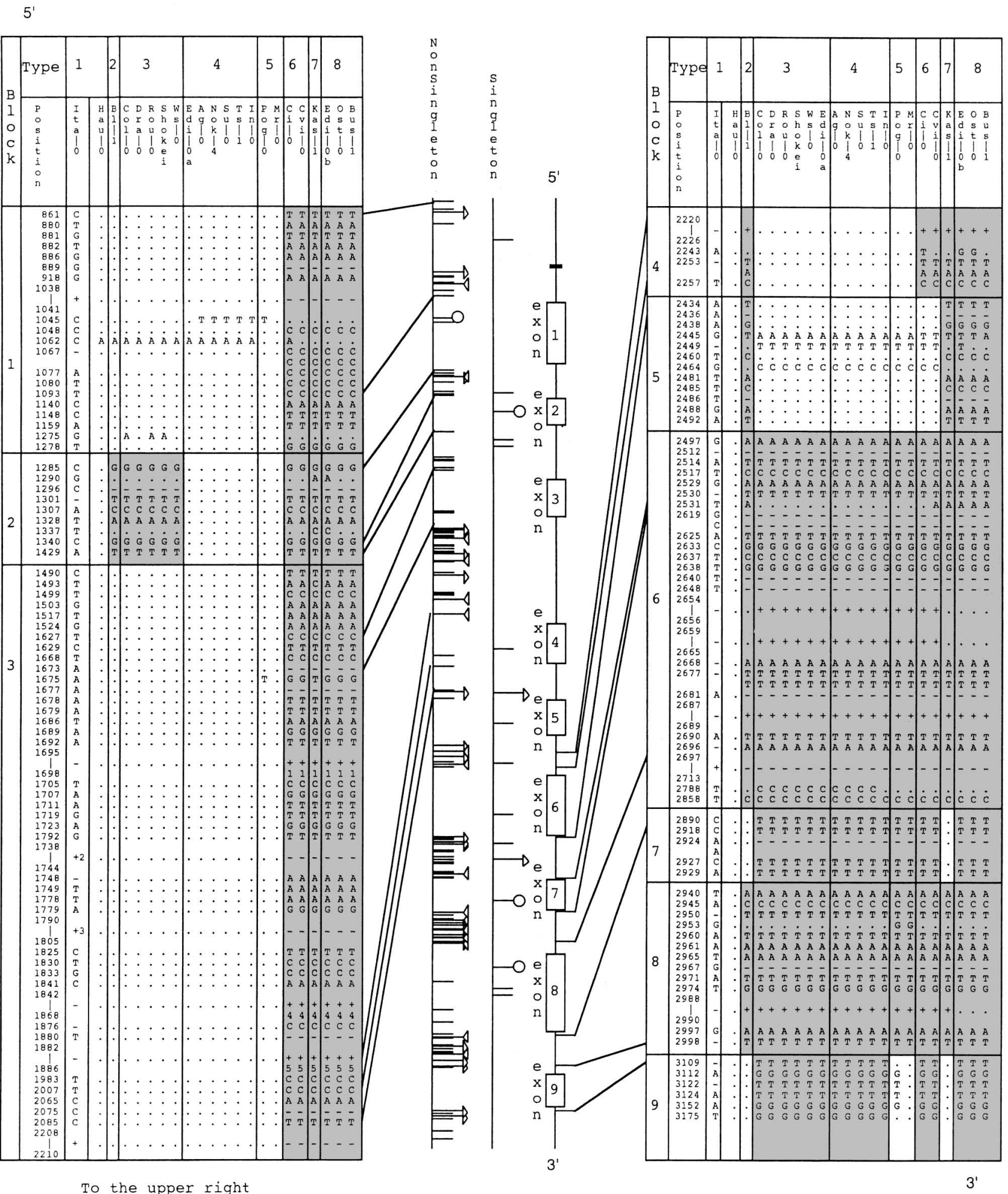

Fig. 1. Polymorphism in the ACL5 region of A. thaliana. The structure of the ACL5 region is shown in the center of figure. Open boxes indicate exons. A black box is the putative auxin-responsive cis-acting elements. DNA variations are shown as vertical bars in the center of the figure. Replacement changes are shown by open circles. Insertion and deletion are shown as triangles on the top of the vertical bar. Nonsingleton variations are summarized on both sides of gene structure, where dots indicate identical to the variation of the Ita- 0 sequence. Insertions and deletions are shown as + and - respectively. +1: AAAA, +2: AGTGCAC, +3: CTTGTTTATTATTATA, +4: TACAAA CATAGTTTTGAATTGTC, +5 TATTTT. + +4 was an insertion caused by duplication. 
(Amersham/Pharmacia Biotech, Piscataway, N.J.). To eliminate PCR artifacts, we mixed the three plasmid clones at almost the same molarity. Sequencing primers were designed at $~ 500$-bp intervals. Newly determined sequences were deposited in the DDBJ databank under accession numbers AB076723-AB076744.

Data Analysis. Including the published sequence of ecotype Columbia, a total of 21 ecotypes of A. thaliana were analyzed. In addition, since Edi-0 was heterozygous in the ACL5 region and had different sequence types, each allele was treated separately and designated Edi- 0 a and Edi- 0 b, respectively. The region analyzed is located between sites 131 and 3021 in the coordinates of the ACL5 sequence of the Columbia ecotype. The DnaSP program version 3.50 (Rozas and Rozas, 1999) was used to analyze intra- and inter-specific variations. Nucleotide diversity $(\pi)$ and $\theta(4 \mathrm{Ne} \mu$ : Watterson, 1975) were estimated after excluding indels. Tajima's test (Tajima, 1989), $\mathrm{Fu}$ and Li's test ( $\mathrm{Fu}$ and $\mathrm{Li}, 1993$ ), MK test (McDonald and Kreitman, 1991) and HKA test (Hudson et al., 1987) were conducted to investigate the departure from neutrality. The run statistic $\left(\mathrm{K}_{R}\right)$, the Kolmogorov-
Smirnov statistics $\left(\mathrm{D}_{K S}\right)$, the maximum sliding $G$ statistics $\left(\mathrm{G}_{\max }\right)$ and the Mean sliding $\mathrm{G}$ statistics $\left(\mathrm{G}_{\min }\right)$ were used to examine whether the ratio of polymorphism to divergence is uniform across a region of the DNA sequence, using the program DNA slider (McDonald, 1996, 1998).

\section{RESULTS}

The pattern of DNA polymorphism in the ACL5 region of $A$. thaliana. A total of 155 nucleotide polymorphisms (110 sites and 45 indels) were detected, of which 139 (96 sites and 43 indels) were found more than once in the samples (Fig. 1). This frequency of non-singletons (90\%: Fig. 2) is extremely high, compared to other genes studied before, where singletons are generally in excess. In the coding region, there were 17 polymorphic sites (13 synonymous sites and 4 nonsynonymous sites) and no insertions or deletions. None of the four nonsynonymous changes was non-conservative with respect to physicochemical differences, according to Miyata et al. (1979). The introns had 115 nucleotide variants (76 sites and 40 indels), of which 71 polymorphic sites and 38

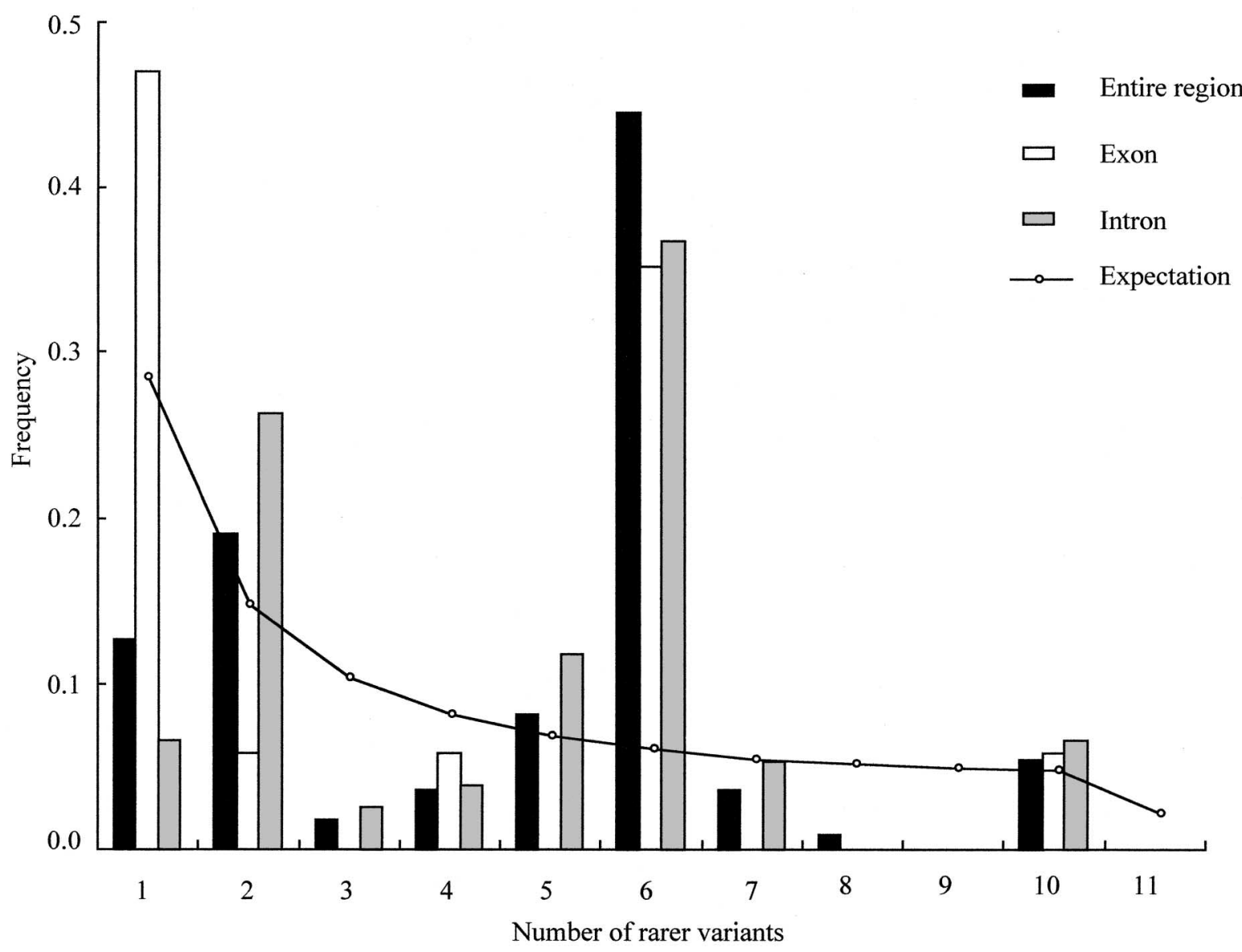

Fig. 2. Frequency spectrum of polymorphic nucleotides of the entire region, exon and intron in the ACL5 region. The expectation was calculated according to Tajima (1989). 


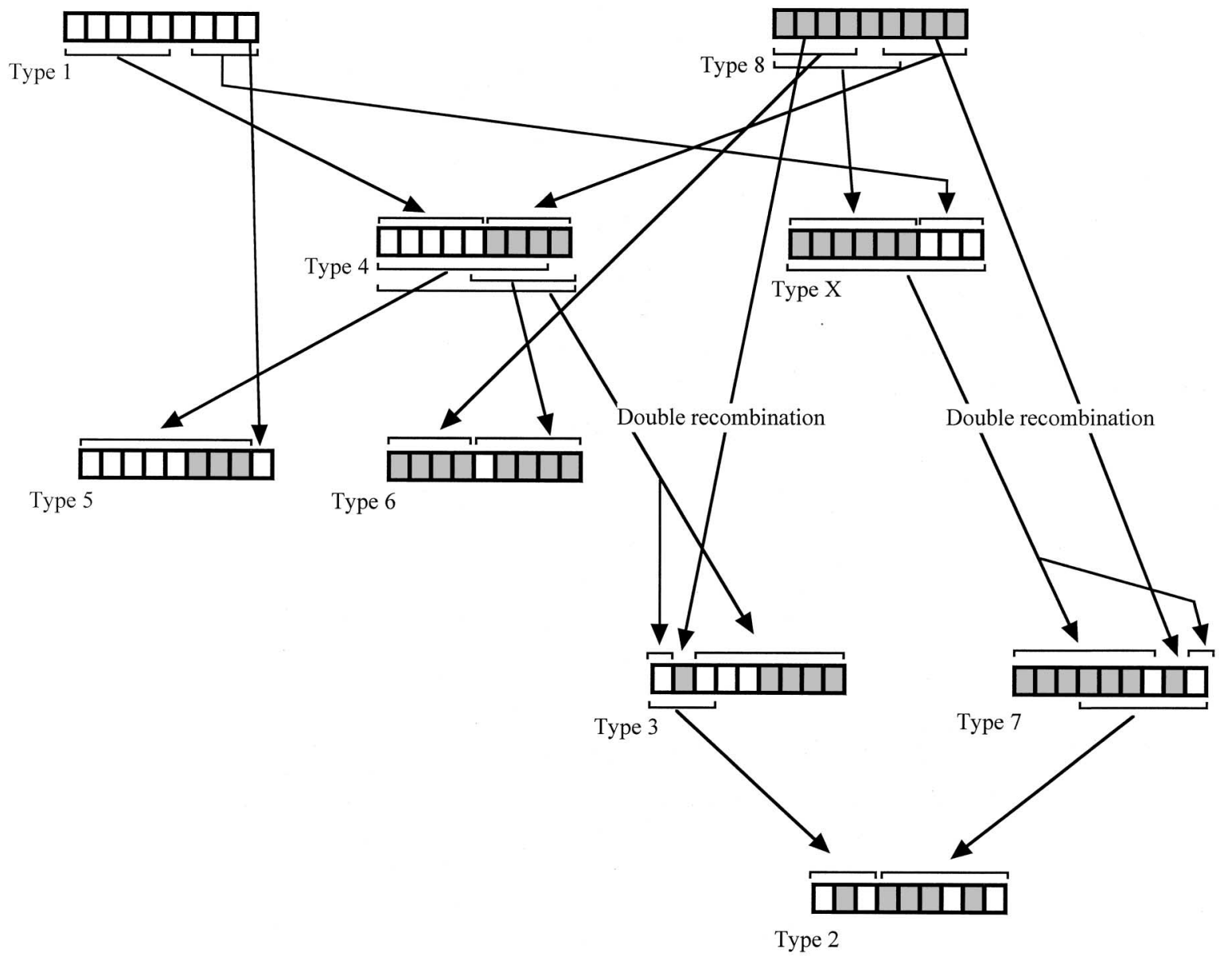

Fig. 3. Diagram of recombination events for the six recombinant sequence types detected in this study. Each block is that shown in the figure 1. Type $\mathrm{X}$ is a hypothetical recombinant.

indels were non-singleton (Fig. 2). In the $5^{\prime}$ upstream region, there were 13 polymorphic sites and 3 indel polymorphisms, but no DNA polymorphisms were detected in a $80 \mathrm{bp}$ region encompassing the two putative auxinresponsive cis-acting elements and TATA Box. The conservativeness of this regulatory region suggests strong selective constraint on auxin-induced ACL5 expression.

It is clear that there is dimorphism in the ACL5 region (Fig. 1). From the distribution of the polymorphic sites, eight distinct sequence types can be defined (Fig. 1). Based on the partition pattern of ecotypes, the ACL5 region can be divided into nine blocks. Since sequence types 1 and 8 were the most divergent, these two sequence types could be assumed to be parental. The rest could be recombinants between sequence types 1 and 8. Assuming a hypothetical recombinant sequence type $\mathrm{X}$ and double recombinations in the history in the ACL5 region of $A$. thaliana, the other six sequence types could be related by seven intragenic recombinations (Fig. 3).

The times of recombination events were estimated by using the number of nucleotide differences between recombinant and parental sequence types as described for the Adh region of $A$. thaliana in Innan et al (1996).
Assuming that the nucleotide mutation rate per site per year is $10^{-9}$ as in Innan et al. (1996), we estimated that recombinant sequence types were generated $0.23 \sim 0.68$ Myr ago. In contrast, the two parental sequence types were estimated to have diverged 20.92 Myr ago, the estimated times above indicates that intragenic recombinations in the ACL5 region had occurred only very recently and in a short interval. The rapid occurrence of recombinations in ACL5 was not observed in Adh, where four intragenic recombinations took place in an equal interval of about one Myr (Innan et al. 1996). Reason for the difference in recombination frequency in the two loci is not clear.

The level of nucleotide variations in the $A C L 5$ region. Nucleotide diversity $(\pi)$ in the entire ACL5 region was 0.016 (Table 2), which is one of the highest known among $A$. thaliana loci. Among different regions of $A C L 5$, it is noted that the introns had the highest nucleotide diversity, and that the replacement variation is very low. Here, the level of variation in the introns of ACL5 was almost twice as high as for synonymous sites in the exons (Table 2). To examine the relationship 
K. YOSHIDA et al.

Table 2. Summary of thr intraspecific DNA variation of ACL5 in A. thaliana.

\begin{tabular}{lcrcrcc}
\hline \hline & \#of sites & \multicolumn{1}{c}{$\mathrm{S}$} & $\pi$ & $\theta$ & Tajima's D & Fu and Li's D* \\
\hline Entire region & 2341 & 110 & 0.016 & 0.014 & $0.631 \mathrm{NS}$ & $0.976 \mathrm{NS}$ \\
Silent & 1407.8 & 106 & 0.025 & 0.021 & $0.709 \mathrm{NS}$ & $1.118 \mathrm{NS}$ \\
Coding region & 1017 & 17 & 0.004 & 0.005 & $-0.991 \mathrm{NS}$ & $-0.930 \mathrm{NS}$ \\
Synonymous & 222.81 & 13 & 0.016 & 0.016 & $-0.029 \mathrm{NS}$ & $-0.503 \mathrm{NS}$ \\
Replacement & 794.19 & 4 & 0.001 & 0.001 & $-1.047 \mathrm{NS}$ & $-1.764 \mathrm{NS}$ \\
Noncoding region & 1324 & 93 & 0.024 & 0.022 & $0.808 \mathrm{NS}$ & $1.346 \mathrm{NS}$ \\
5' flanking & 256 & 13 & 0.021 & 0.014 & $1.570 \mathrm{NS}$ & $1.088 \mathrm{NS}$ \\
Intron & 976 & 76 & 0.029 & 0.026 & $0.580 \mathrm{NS}$ & $1.317 \mathrm{NS}$ \\
3' flanking & 92 & 4 & 0.019 & 0.012 & $1.446 \mathrm{NS}$ & $1.096 \mathrm{NS}$ \\
\hline
\end{tabular}

NS: not significant

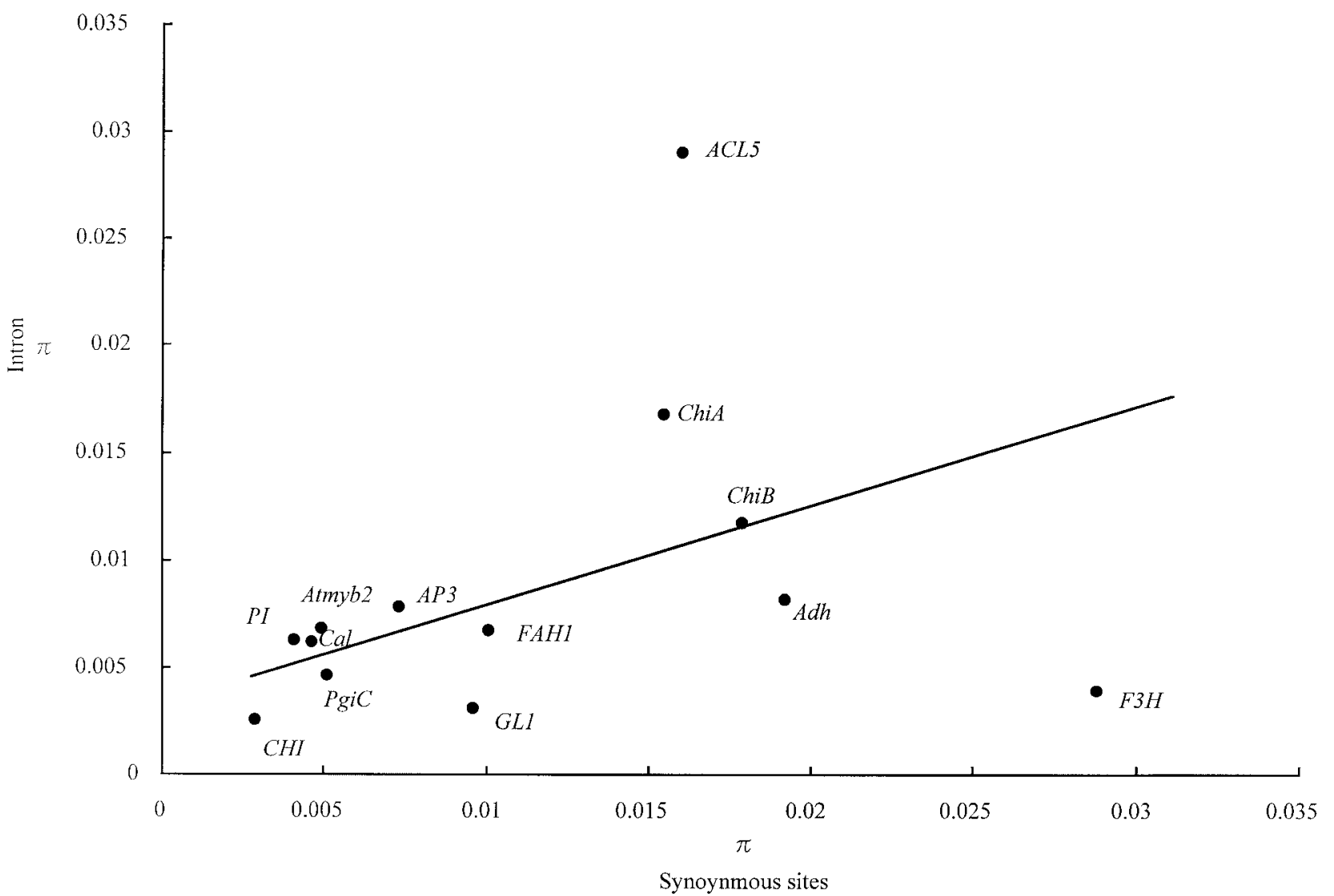

Fig. 4. Correlation of nucleotide diversity between synonymous sites in exon and intron. Regression line is for 11 gene regions except the ACL5 and F3H regions. The square of the correlation coefficient is 0.450 .

between levels of nucleotide variations between exon (synonymous sites) and intron, the nucleotide diversities were plotted for ACL5 and other loci of A. thaliana (Fig. 4). There is no correlation between the two regions. The level of synonymous variation of ACL5 exon was intermediate among other loci in A. thaliana, while the introns of ACL5 had an exceptionally high nucleotide diversity.

$\chi^{2}$ tests were conducted to examine the difference in the number of segregating sites between the exons and introns. When all the sites were considered, the number of segregating sites in the introns (76) was significantly higher than in the exon $\left(17, \chi_{\mathrm{df}=1}^{2}=39.91, P<\right.$ 0.0001). However, no difference was detected in the number of segregating silent sites between the exon (13) and intron $\left(76, \chi_{\mathrm{df}=1}^{2}=0.93, N S\right)$. Interestingly, the number of nonsingletons in the intron (71) was significantly higher than the number of synonymous sites in the 
exon $\left(5, \chi_{\mathrm{df}=1}^{2}=7.24, P<0.05\right)$. These results indicated that the higher nucleotide diversity in the intron than in the exon (synonymous sites) is caused by the large number of nonsingletons in the intron.
Tests of neutrality on the ACL5 region. None of the neutrality tests gave significant results for DNA variations in the ACL5 region (Table 2). The test statistics were negative for the coding region, even for synonymous sites. This result is consistent with the observations

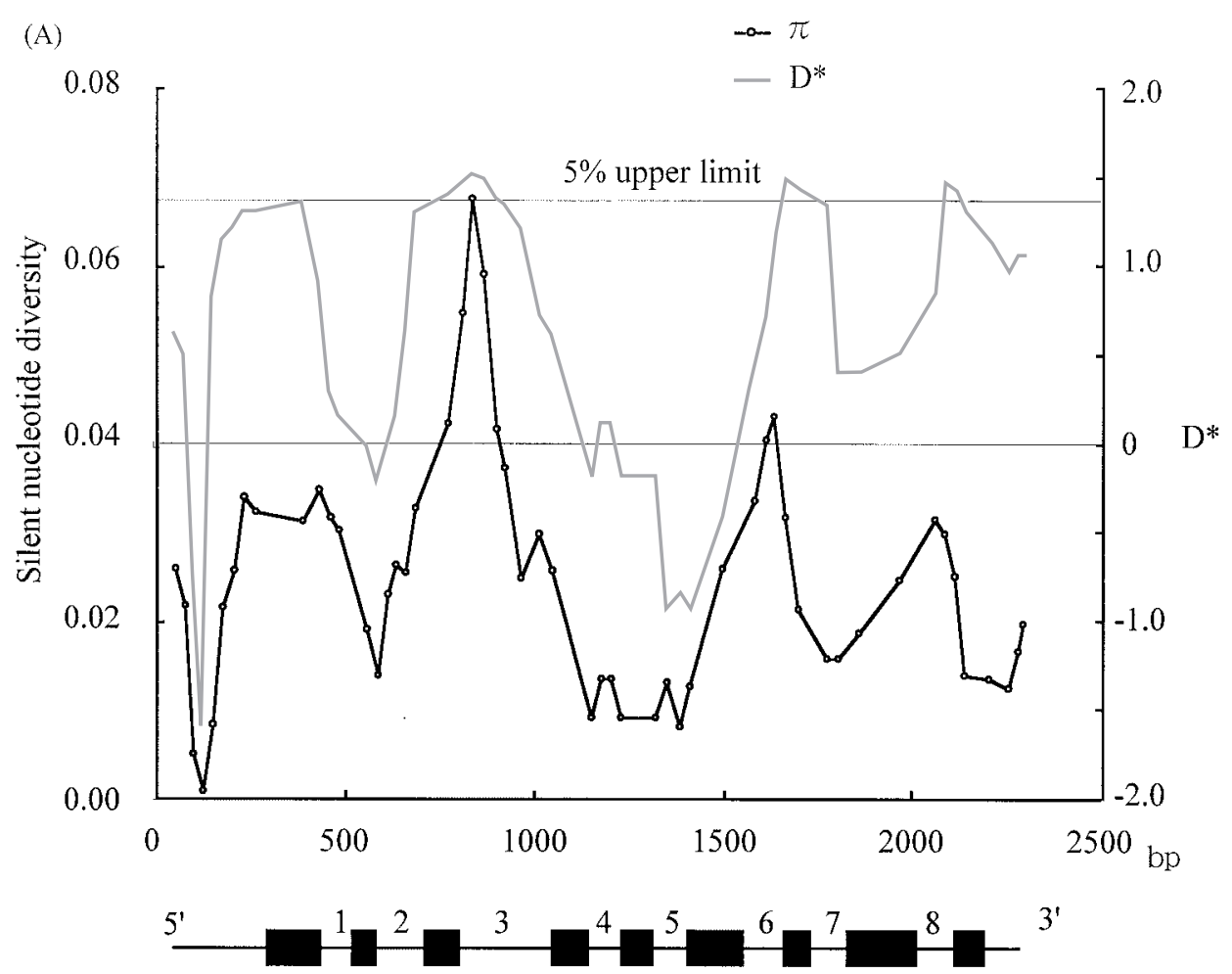

(B)

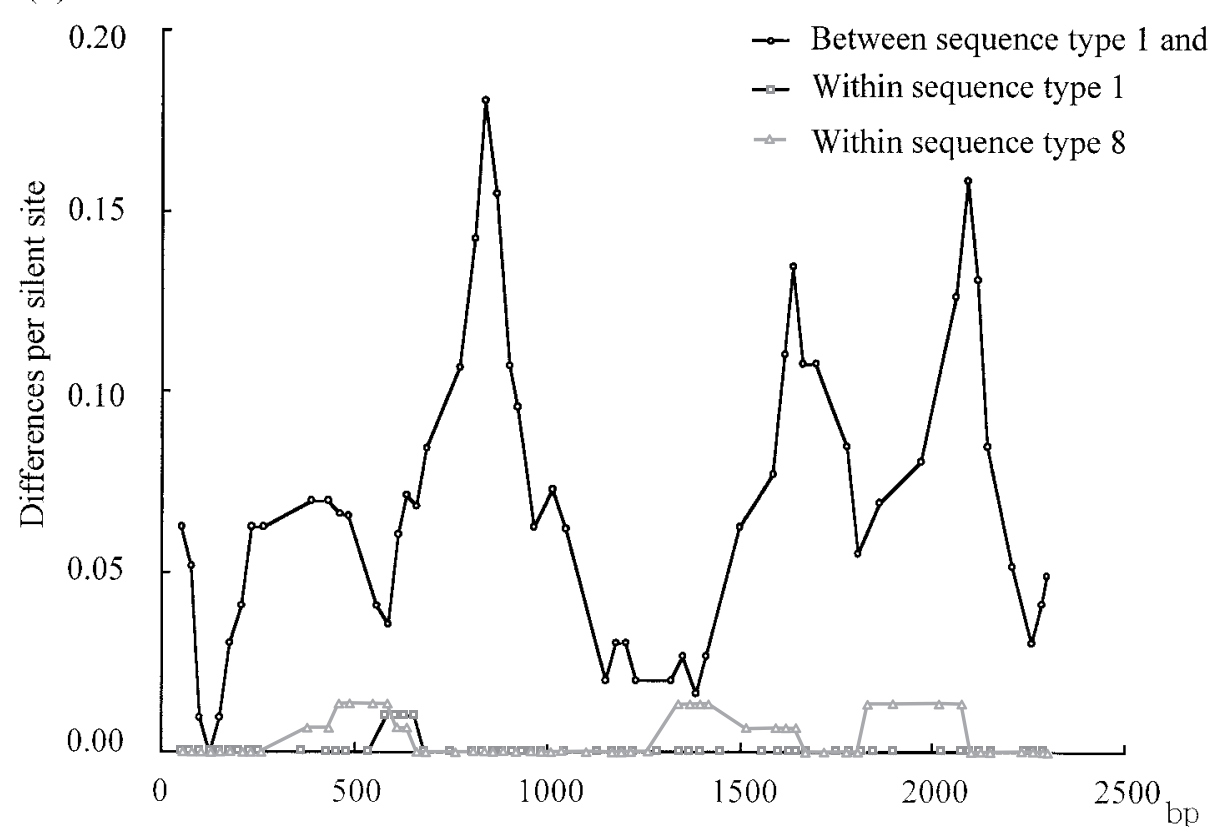

Fig. 5. Sliding window plot for the ACL5 region of A. thaliana. (A) Nucleotide diversity of silent sites and Fu and Li's $\mathrm{D}^{*}$ for all the ecotypes are shown. Confidence intervals for 5\% and 10\% significance levels of a two-tailed Fu and Li's test are shown for a sample size of 22. (B) Nucleotide diversity for sequence types 1 and 8 is shown. In all cases, the window size is $100 \mathrm{bp}$, step size is $25 \mathrm{bp}$, and unit is silent site. 
that the level of replacement variation was low, and that the coding region had fewer nonsingleton sites than the intron, suggesting purifying selection on the coding region. Although an extremely high level of nucleotide variation was detected in the intron, no significance in the neutrality tests was detected for the intron.

To examine the change in the level of polymorphism among functionally different regions of ACL5, the sliding window analysis, using $\mathrm{Fu}$ and Li's $\mathrm{D}^{*}$ which compares the numbers of singletons and nonsingletons, was conducted (Fig. 5-A). Positive $\mathrm{D}^{*}$ values indicate an excess of nonsingletons. There were high peaks, especially in the introns 3,6 and 8. Fu and Li's D* was significantly positive around the peaks detected in the three introns, although significance was not obtained after the Bonferroni multiple correction.

A $\chi^{2}$ test to examine whether the number of nonsingletons varies over different introns revealed significant heterogeneity in the number of nonsingletons among introns $\left(\chi^{2}=16.15, \mathrm{df}=7 \mathrm{P}<0.01\right)$. This heterogeneity is obviously caused by the excess of nonsingletons in introns 3 , 6 and 8 . Figure 5-B shows sliding window plots of nucleotide variations for the two divergent sequence types 1 and 8 . High peaks in the introns 3,6 and 8 were also

Table 3. Genetic distance of nuclear genes between A. thaliana and A. gemmifera

\begin{tabular}{lccccc}
\hline \hline & $\mathrm{K}$ & $\mathrm{K}$ (intron) & $\mathrm{Ks}$ & $\mathrm{Ka}$ & $\mathrm{Ka} / \mathrm{Ks}$ \\
\hline Acl5 & 0.034 & 0.109 & 0.147 & 0.006 & 0.038 \\
Adh & 0.061 & 0.132 & 0.192 & 0.016 & 0.084 \\
ChiA & 0.057 & 0.108 & 0.139 & 0.033 & 0.241 \\
ChiB & 0.054 & 0.297 & 0.157 & 0.025 & 0.156 \\
PgiC & 0.044 & 0.124 & 0.172 & 0.009 & 0.054 \\
Atmyb2 & 0.073 & 0.119 & 0.233 & 0.033 & 0.141 \\
\hline
\end{tabular}

Ks: Synonymous divergence

Ka: Replacement divergence

Table 4. Summary of HKA test on the ACL5 and other loci

\begin{tabular}{|c|c|c|c|c|c|c|}
\hline \multirow[b]{2}{*}{ Locus } & & \multicolumn{2}{|c|}{ Numver of sites (bp) } & \multicolumn{2}{|c|}{ Segregating sites per site } & \\
\hline & & Within & between & Within & Between & \\
\hline \multirow[t]{4}{*}{$A C L 5(\mathrm{n}=22)$} & Exon & 1017 & 1017 & 0.017 & 0.033 & \\
\hline & Syn & 222.8 & 222.8 & 0.058 & 0.134 & \\
\hline & Rep & 794.2 & 795.2 & 0.005 & 0.006 & \\
\hline & Intron & 836 & 770 & 0.091 & 0.101 & \\
\hline \multirow[t]{4}{*}{ vs $A d h(\mathrm{n}=17)$} & Exon & 1137 & 1137 & 0.017 & 0.032 & \\
\hline & Syn & 252.7 & 253 & 0.051 & 0.119 & $0.009 \mathrm{NS}$ \\
\hline & Rep & 884.3 & 884 & 0.009 & 0.016 & $0.488 \mathrm{NS}$ \\
\hline & Intron & 540 & 495 & 0.037 & 0.128 & $2.940 \mathrm{NS}$ \\
\hline \multirow[t]{4}{*}{ vs $C h i A(\mathrm{n}=17)$} & Exon & 906 & 906 & 0.049 & 0.054 & $1.027 \mathrm{NS}$ \\
\hline & Syn & 204 & 205.2 & 0.123 & 0.126 & $1.528 \mathrm{NS}$ \\
\hline & Rep & 702 & 700.8 & 0.027 & 0.033 & $0.000 \mathrm{NS}$ \\
\hline & Intron & 426 & 407 & 0.059 & 0.099 & $0.262 \mathrm{NS}$ \\
\hline \multirow[t]{4}{*}{ vs $C h i B(\mathrm{n}=17)$} & Exon & 1008 & 1008 & 0.022 & 0.052 & $0.023 \mathrm{NS}$ \\
\hline & Syn & 240.3 & 240.3 & 0.071 & 0.141 & $0.098 \mathrm{NS}$ \\
\hline & $\operatorname{Rep}$ & 767.7 & 767.7 & 0.005 & 0.024 & $1.947 \mathrm{NS}$ \\
\hline & Intron & 427 & 190 & 0.056 & 0.245 & $6.069 *$ \\
\hline \multirow[t]{4}{*}{ vs $P g i C(\mathrm{n}=21)$} & Exon & 1667 & 1656 & 0.018 & 0.042 & $0.037 \mathrm{NS}$ \\
\hline & Syn. & 388.2 & 383.6 & 0.041 & 0.153 & $0.560 \mathrm{NS}$ \\
\hline & Rep & 1288.8 & 1269.4 & 0.012 & 0.010 & $0.068 \mathrm{NS}$ \\
\hline & Intron & 2837 & 2823 & 0.032 & 0.114 & $6.609 *$ \\
\hline
\end{tabular}

$* \mathrm{P}<0.05 ; \mathrm{NS}$ : not significant 
detected. When the sequence types 1 and 8 were considered separately, the peaks disappeared. This result indicates that these peaks in introns are caused by difference between the divergent sequence types.

The divergence in the $A C L 5$ region between $A$. thaliana and A. gemmifera. ACL5 has the lowest divergence between $A$. thaliana and A. gemmifera in both the coding and intron regions among all the genes compared (Table 3). Corresponding with the low replacement polymorphism (Table 2), replacement divergence of $A C L 5$ was also the lowest, suggesting again purifying selection on the amino acid substitutions in the coding region of ACL5.

HKA tests were applied to examine the correlation between polymorphism in A. thaliana and divergence between the two species (Table 4). No significant deviations were detected for the exons. On the other hand, the HKA test for intron indicated that ACL5 was significantly different in comparison with $C h i B$ or $P g i C$, and revealed a marginally significant difference compared with Adh. In these cases of HKA test, obviously the high level of polymorphism in intron of ACL5 contributed to the significance (and nearly significance), although signif- icance was not obtained after the Bonferroni multiple correction. It can be concluded that the ACL5 intron has a significantly higher ratio of polymorphism and divergence compared to those of introns of other loci in $A$. thaliana. The MK tests found no significant results.

Figure 6 shows a sliding window plot for silent divergence between the two species. High peaks of divergence in the three introns $(3,6$, and 8$)$ were detected, as for polymorphism. The tests of heterogeneity in the ratio of silent polymorphism to divergence (McDonald, 1996, 1998) were applied to the ACL5 region, but they did not reveal any significance. It can be concluded that the distribution of divergence was compatible with that of polymorphism. When sequence types 1 and 8 were analyzed separately, high peaks of divergence in the introns, especially intron 3 , were still found (data not shown). Therefore, it can be concluded that the peaks of divergence in the introns were not due to the dimorphic variation.

The $5^{\prime}$ upstream region containing putative auxinresponsive cis-acting elements and TATA box is conserved between A. thaliana and A. gemmifera. Although 18 substitutions were detected $(7.2 \%)$ in the entire $5^{\prime}$ region, in the 80 bp region with the two regulatory ele-

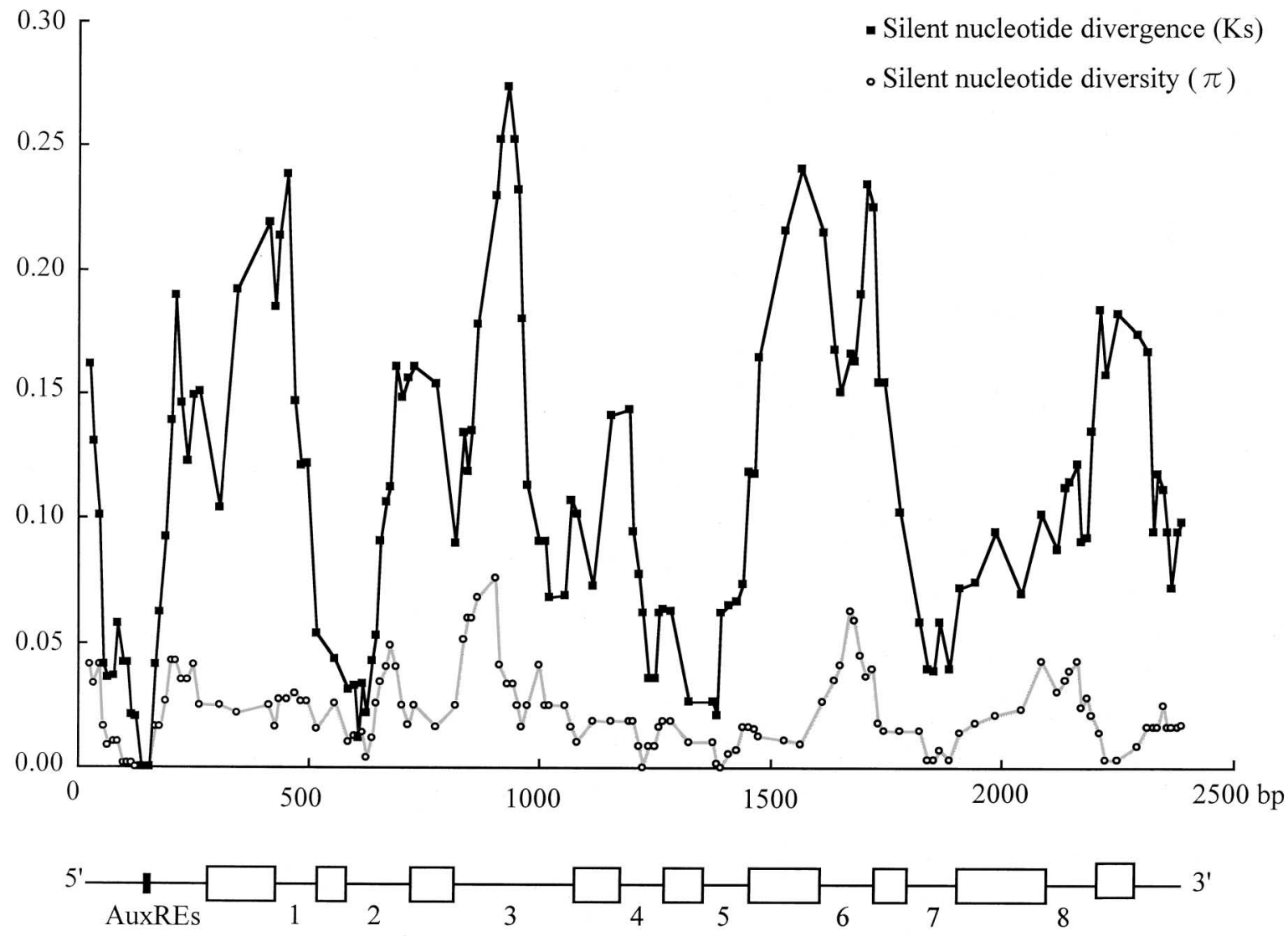

Fig. 6. Sliding window plot for the ACL5 region between A. thaliana and A. gemmifera. Open boxes indicate exons, and the black box indicates the putative auxin-responsive cis-elements (AuxREs). Window size is $50 \mathrm{bp}$, step size is $10 \mathrm{bp}$. 
ments and TATA Box, only one site substitution was detected. Around the region of auxin regulation, no peak of variation was detected in either polymorphism or divergence (Fig. 6). These results suggest that regulation of auxin-induced ACL5 expression is essential for both species.

\section{DISCUSSION}

DNA polymorphism in the $A C L 5$ region of $A$. thaliana. As in some other nuclear loci of A. thaliana (11 out 13 loci studied so far), the ACL5 region was found to be dimorphic. Intragenic recombinations in the ACL5 region occurred more recently and in a shorter interval than those in the Adh region. Although there may be some genetic mechanisms underlying this difference, a possible explanation would be subpopulation structure in the ancient $A$. thaliana population before spreading over the world. Supposing that the ancient population consisted of many isolated subpopulations, differentiation of divergent sequence types in each of the two regions would proceed independently in different isolated populations. Fused populations could be segregating in Adh but not in ACL5, and vice versa. Probably, fusion of isolated populations containing different sequence types occurred only recently for ACL5. The exact reason for the difference in intragenic recombination in the two regions is not clear.

This study demonstrated that the nucleotide variation in the ACL5 region is one of the highest among $A$. thaliana nuclear genes, which is due to the unusually high level of variation in the introns. Also, in this region the number of nonsingletons was very high, unlike other regions studied in this species. Especially the introns had significantly more nonsingletons than the coding region (synonymous sites). The HKA test statistically confirmed that the level of nucleotide polymorphism in introns of ACL5 was high.

Despite the high level of variation in the entire region, the 5 ' flanking region containing putative auxin-responsive cis-acting elements and TATA box is highly conserved, even in the interspecific comparison with $A$. gemmifera. This result indicated that auxin regulation in ACL5 is essential in these two species. To clarify if the low level of variation in the auxin-regulatory region is gene- or genus-specific, DNA variation in other auxinregulated genes must be analyzed in the future.

High level of nucleotide variation in the introns of ACL5 region. One possible explanation for high level of polymorphism is a high mutation rate. However, divergence between A. thaliana and A. gemmifera was not unusually high compared with other loci. If the mutation rate is related to the high level of polymorphism, the mutation rate in this region must have been elevated in the A. thaliana lineage after split of the two species. The observation that the coding region of ACL5 had the same levels of polymorphism as those of other loci could be explained by purifying selection. Since introns could be subjected to less stringent selection pressure than the coding region, a high mutation rate would be reflected directly on the high level of the variation in the introns. Coincidentally, the ChiA locus located on the short arm of chromosome 5, as the ACL5 locus, also has a high level of nucleotide variation (Kawabe et al., 1997). Regional hotspots of nucleotide mutation might exist in the A. thaliana genome.

The other possibility for a high level of polymorphism is a balancing selection. There are high peaks of nucleotide variation in three introns, which disappear when the two divergent sequence types were analyzed separately. This kind of pattern of DNA variation was invoked as an evidence of balancing selection, such as overdominance, for Adh of Drosophila melanogaster (Hudson and Kaplan, 1988), and Adh and PgiC of A. thaliana (Innan et al., 1996; Kawabe et al., 2000; Miyashita, 2001). In these previous cases, peaks of nucleotide variation were clearly associated with replacement sites in the exon region, which are responsible for allozyme variation of these genes. Here, it seems difficult to assume natural selection, since the peaks were found in introns, which do not influence protein product. However, it has been shown that DNA variation in intron influences the enzyme activity of ADH in Drosophila melanogaster (Laurie et al., 1991), where an indel polymorphism detected in the first intron is related to a change in enzyme concentration, affecting the activity. In intron 3 of $A C L 5$, where the highest peak of nucleotide variation was detected, a duplicated sequence was found polymophic between the two divergent sequence types. This indel might be related to ACL5 expression, as the indel in Adh of D. melanogaster. At this moment, it is not possible to specify the molecular basis of balancing selection for ACL5. Further molecular analyses on enzyme activity and gene expression are necessary to specify if the high peaks of nucleotide variation in introns are related to some form of natural selection or not.

Authors express their thanks to R. Terauchi for comments on this paper. This is contribution \#574 from the Laboratory of Plant Genetics, Graduate School of Agriculture, Kyoto University.

\section{REFERENCES}

Aguadé, M. (2001) Nucleotide sequence variation at two genes of the phenylpropanoid pathway, the $F A H 1$ and $F 3 H$ genes, in Arabidopsis thaliana. Mol. Biol. Evol. 18, 1-9.

Caicedo, A. L., Schaal, B. A., and Kunkel, B. N. (1999) Diversity and molecular evolution of the RPS2 resistance gene in Arabidopsis thaliana. Proc. Natl. Acad. Sci. U.S.A. 96, 302306.

Gilroy, S., and Trewavas, A. (2001) Signal processing and transduction in plant cells: the end of the beginning? Nat. Rev. 
Mol. Cell Biol. 2, 307-314.

Fu, Y. X., and Li, W. H. (1993) Statistical tests of neutrality of mutations. Genetics 133, 693-709.

Hanzawa, Y., Takahashi, T., Michael, A. J., Burtin, D., Long, D., Pineiro, M., Coupland, G., and Komeda, Y. (2000) ACAULIS5, an Arabidopsis gene required for stem elongation, encodes a spermine synthase. The EMBO J. 19, $4248-4256$.

Hauser, M. T., Harr, B., and Schlötterer, C. (2001) Trichome distribution in Arabidopsis thaliana and its close relative Arabidopsis lyrata: molecular analysis of the candidate gene GLABROUS1. Mol. Biol. Evol. 18, 1754-1763.

Hudson, R. R., and Kaplan, N. L. (1988) The coalescent process in models with selection and recombination. Genetics 120, 831-840.

Hudson, R. R., Kreitman, M., and Aguadé, M. (1987) A test of neutral molecular evolution based on nucleotide data. Genetics 116, 153-159.

Innan, H., Tajima, F., Terauchi, R., and Miyashita, N. T. (1996) Intragenic recombination in the Adh locus of the wild plant Arabidopsis thaliana. Genetics 143, 1761-1770.

Kawabe, A., Innan, H., Terauchi, R., and Miyashita, N. T. (1997) Nucleotide polymorphism in the acidic chitinase locus (ChiA) region of the wild plant Arabidopsis thaliana. Mol. Biol. Evol. 14, 1303-1315.

Kawabe, A., and Miyashita, N. T. (1999) DNA variation in the basic chitinase locus $(C h i B)$ region of the wild plant Arabidopsis thaliana. Genetics 153, 1445-1453.

Kawabe, A., Yamane, K., and Miyashita, N. T. (2000) DNA polymorphism at the Cytosolic Phosphoglucose Isomerase (PgiC) locus of the wild plant Arabidopsis thaliana. Genetics 156, $1339-1347$

King, J. J., and Stimart, D. P. (1998) Genetic analysis of variation for auxin-induced adventitious root formation among eighteen ecotypes of A. thaliana L. Heynh. J. Hered. 89, 481-487.

Kuittinen, H., and Aguadé, M. (2000) Nucleotide variation at the CHALCONE ISOMERASE locus in Arabidopsis thaliana. Genetics 155, 863-872.

Laurie, C. C., Bridgham, J. T., and Choudhary, M. (1991) Associations between DNA sequence variation and variation in expression of the Adh gene in natural populations of Drosophila melanogaster. Genetics 129, 489-499.

Leyser O. (2001) Auxin signaling: the beginning, the middle and the end. Curr. Opin. Plant Biol. 4, 382-386.

McDonald, J, H. (1996) Detecting non-neutral heterogeneity across a region of DNA sequence in the ratio of polymorphism to divergence. Mol. Biol. Evol. 13, 253-260.

McDonald, J. H. (1998) Improved tests for heterogeneity across a region of DNA sequence in the ratio of polymorphism to divergence. Mol. Biol. Evol. 15, 377-384.

McDonald, J. H., and Kreitman, M. (1991) Adaptive protein evolution at the Adh locus in Drosophila. Nature 351, 652654.

Miyashita, N. T. (2001) DNA variation in the 5'upstream region of the Adh locus of the wild plants Arabidopsis thaliana and Arabis gemmifera. Mol. Biol. Evol. 18, 164-171.

Miyashita, N. T., Innan, H., and Terauchi, R. (1996) Intra-and interspecific variation of the alcohol dehydrogenase locus region of wild plants Arabis gemmifera and Arabidopsis thaliana. Mol. Biol. Evol. 13, 433-436.

Miyata, T., Miyazawa, S., and Yasunaga, T. (1979) Two types of amino acid substitutions in protein evolution. J. Mol. Evol 12, 219-236.

Moriyama, E. N., and Powell, J. R. (1996) Intraspecific nuclear DNA variation in Drosophila. Mol. Biol. Evol. 13, 261277.

Nei, M., and Li W-S. (1979) Mathematical model for studying genetic variation in terms of restriction endonucleases. Proc. Natl. Acad. Sci. U.S.A. 76, 5269-5273.

Purugganan, M. D., and Suddith, J. I. (1998) Molecular population genetics of the Arabidopsis CAULIFLOWER regulatory gene: Nonneutral evolution and naturally occurring variation in floral homeotic function. Proc. Natl. Acad. Sci. U.S.A. 95, 8130-8134.

Purugganan, M. D., and Suddith, J. I. (1999) Molecular population genetics of floral homeotic loci: Departures from the equilibrium-neutral model at the APETALA3 and PISTILLATA genes of Arabidopsis thaliana. Genetics 151, 839848.

Rozas, J., and Rozas, R. (1999) DnaSP version 3: an integrated program for molecular population genetics and molecular evolution analysis. Bioinformatics 15, 174-175.

Stahl, E. A., Dwyer, G., Mauricio, R., Kreitman, M., and Bergelson, J. (1999) Dynamics of disease resistance polymorphism at the Rpm1 locus of Arabidopsis. Nature 400, 667-671.

Tajima, F. (1989) Statistical method for testing the neutral mutation hypothesis by DNA polymorphism. Genetics 123, $585-595$.

Tajima, F., and Nei, M. (1984) Estimation of evolutionary distance between nucleotide sequences. Mol. Biol. Evol. 1, 269-285.

Walden, R., Cordeiro, A., and Tiburcio, A. F. (1997) Polyamines: Small molecules triggering pathways in plant growth and development. Plant physiol. 113, 1009-1013.

Watterson, G. A. (1975) On the number of segregating sites in genetical models without recombination. Theor. Popul. Biol. 7, 256-276. 